\title{
Cartographie Géotechnique par Deep Learning Approche par Réseaux de Neurones Artificiels
}

\author{
Kasongo wa Mutombo Portance, MSc
}

Université de Lubumbashi, Faculté des Sciences, Département de Géologie, Lubumbashi, République Démocratique du Congo

Kavula Ngoy Elysée, Chercheur-Ingénieur

Institut Supérieur des Techniques Appliquées de Lubumbashi, Département de Génie Civil, Lubumbashi, République Démocratique du Congo

Sow Libasse, Docteur en génie civil de l'INSA Rennes, France

Département Génie Civil, Ecole Supérieure Polytechnique,

Université Cheikh Anta Diop de Dakar, Sénégal

\section{Lunda Ilunga Jean Marie, PhD}

Université de Lubumbashi, Faculté des Sciences, Département de Géologie, République Démocratique du Congo

Doi: 10.19044/esj.2019.v15n12p233 URL:http://dx.doi.org/10.19044/esj.2019.v15n12p233

\section{Résumé}

En géotechnique, les ingénieurs utilisent plusieurs systèmes de classification des géomatériaux en affectant à chaque catégorie par similitude de leurs propriétés physiques et/ou mécaniques un code. Pour réaliser la classification de chaque système, ils recourent souvent à la méthode algorithmique qui consiste à comparer chaque paramètre aux seuils fixés. Cette méthode est envisageable quand des données à traiter ne sont pas massives. L'objectif principal est de proposer une nouvelle méthode de classification des géomatériaux applicable à tous le cas des figures. La méthodologie adoptée est celle de l'intelligence artificielle ; l'approche par « deep learning » qui est la plus adaptée dans des problèmes de classification. Pour implémenter le modèle neuronal, l'architecture du perceptron multicouche a été retenue . Elle nécessite la connaissance des données d'entrée (inputs) et celles de sorties (outputs) avec ou sans couches cachées. En comparant les résultats obtenus à l'aide de l'approche algorithmique et ceux de la méthode neuronale, plusieurs points communs sont identifiés (à 86\%). La méthode par réseau de neurones a permis de discriminer certaines classes passées inaperçues avec la classification algorithmique. Cette méthode conduit à des résultats très interessants aussi en se basant seulement sur des photographies numériques prises lors d'une campagne géotechnique. Dans ce dernier cas, les données d'entrée sont constituées des pixels. 
Mots-clés : Classification des Géomatériaux, Intelligence Artificielle, Deep Learning, Perceptron Multicouche, Keras

\title{
Cartographie Géotechnique par Deep Learning Approche par Réseaux de Neurones Artificiels
}

\author{
Kasongo wa Mutombo Portance, MSc \\ Université de Lubumbashi, Faculté des Sciences, \\ Département de Géologie, Lubumbashi, République Démocratique du Congo \\ Kavula Ngoy Elysée, Chercheur-Ingénieur \\ Institut Supérieur des Techniques Appliquées de Lubumbashi, Département \\ de Génie Civil, Lubumbashi, République Démocratique du Congo \\ Sow Libasse, Docteur en génie civil de l'INSA Rennes, France \\ Département Génie Civil, Ecole Supérieure Polytechnique, \\ Université Cheikh Anta Diop de Dakar, Sénégal \\ Lunda Ilunga Jean Marie, PhD \\ Université de Lubumbashi, Faculté des Sciences, \\ Département de Géologie, République Démocratique du Congo
}

\begin{abstract}
In geotechnical engineering, engineers use several systems for classifying geomaterials by assigning each category similarity in their physical and/or mechanical properties to a code. To achieve the classification of each system, they often resort to the algorithmic method which consists of comparing each parameter to the set thresholds. This method is conceivable when the data to be processed are not massive. The main objective is to propose a new method of classifying geomaterials applicable to all cases of figures. The methodology adopted is that of artificial intelligence; the "deep learning" approach which is the most appropriate in classification problems. To implement the neural model, the architecture of the multilayer perceptron has been retained. It requires knowledge of input data and output data with or without hidden layers. By comparing the results obtained using the algorithmic approach and those of the neuronal method, several common points are identified $(86 \%)$. The neural network method made it possible to discriminate certain classes that have not been noticed with the algorithmic classification. This method leads to very interesting results too based only on
\end{abstract}


digital photographs taken during a geotechnical investigation. In the latter case, the input data consists of pixels.

Keywords: Geomaterials Classification, Artificial Intelligence, Deep Learning, Multilayer Perceptron, Keras

\section{Introduction}

Les techniques routières dans leur globalité font appel à des matériaux capables de reprendre et de repartir convenablement les charges extérieures vers les sols support. Pour ce qui est des matériaux composant l'infrastructure elle-même, de nombreux travaux scientifiques ont été faits pour une maitrise plus ou moins parfaite de leur comportement mécanique. On en distingue deux : les granulats naturels issus de carrières d'extraction et les matériaux alternatifs tels que les mâchefers d'incinération de déchets non dangereux qui ont fait l'objet de publications récentes (Sow et al., 2017a, 2017b, 2018a, 2018b, 2018c, 2018d, 2018e, 2019a, 2019b, 2019c, entre autres). Cependant, le comportement mécanique de l'infrastructure est en corrélation avec celui du ou des matériaux constituant le sol support. Pour cela, des efforts ont été faits pour mieux maitriser le comportement de ces sols. Les systèmes de classification des sols sont donc nés du besoin des ingénieurs civils de disposer des renseignements suffisamment fiables sur le comportement des sols pour pouvoir prendre des décisions rapides et efficaces, surtout dans les domaines de construction routière, des infrastructures, des pistes d'atterrissage ou des barrages.

Il existe de très nombreux systèmes de classification des géomatériaux. Celle utilisée dans cet article pour illustrer la méthode est la classification AASHTO (American Association for State Highway Transportation Official). Ce système est très documenté et présente peu des paramètres pour la réaliser (USDA, 1987 ; Das et al, 2012).

La mise en place d'algorithmes de classification demande de temps considérables et une maitrise de la programmation dans la phase d'implémentation. Ces algorithmes ont servi par exemple à classifier les sols de la ville de Lubumbashi (Kasongo, 2017a, 2017b, 2018). Cet inconvénient a poussé à monter une architecture de réseaux de neurones artificiels utilisées dans un délai très bref, avec une performance acceptable.

Les réseaux artificiels permettent d'aborder sous des nouveaux angles les problèmes de perception, de mémoire, d'apprentissage et de raisonnement. Grâce à leur traitement parallèle de l'information et à leurs mécanismes inspirés de cellules nerveuses (neurones), ils infèrent des propriétés émergentes permettant de solutionner des problèmes qualifiés de complexes dans le passé (Parizeau, 2004). Cette complexité était liée à des mémoires faibles des anciens ordinateurs. 
Plusieurs architectures des réseaux artificiels sont utilisées en pratique en fonction des problèmes à résoudre. Pour ce problème de classification des géomatériaux, c'est le perceptron multicouche qui a été retenu.

Les données utilisées pour valider la méthode ont été récoltées au voisinage de l'aéroport international de la Luano. Ce site est localisé au NordEst du centre-ville de Lubumbashi dans le sous bassin versant de la rivière Luano comme montré sur la figure 1.

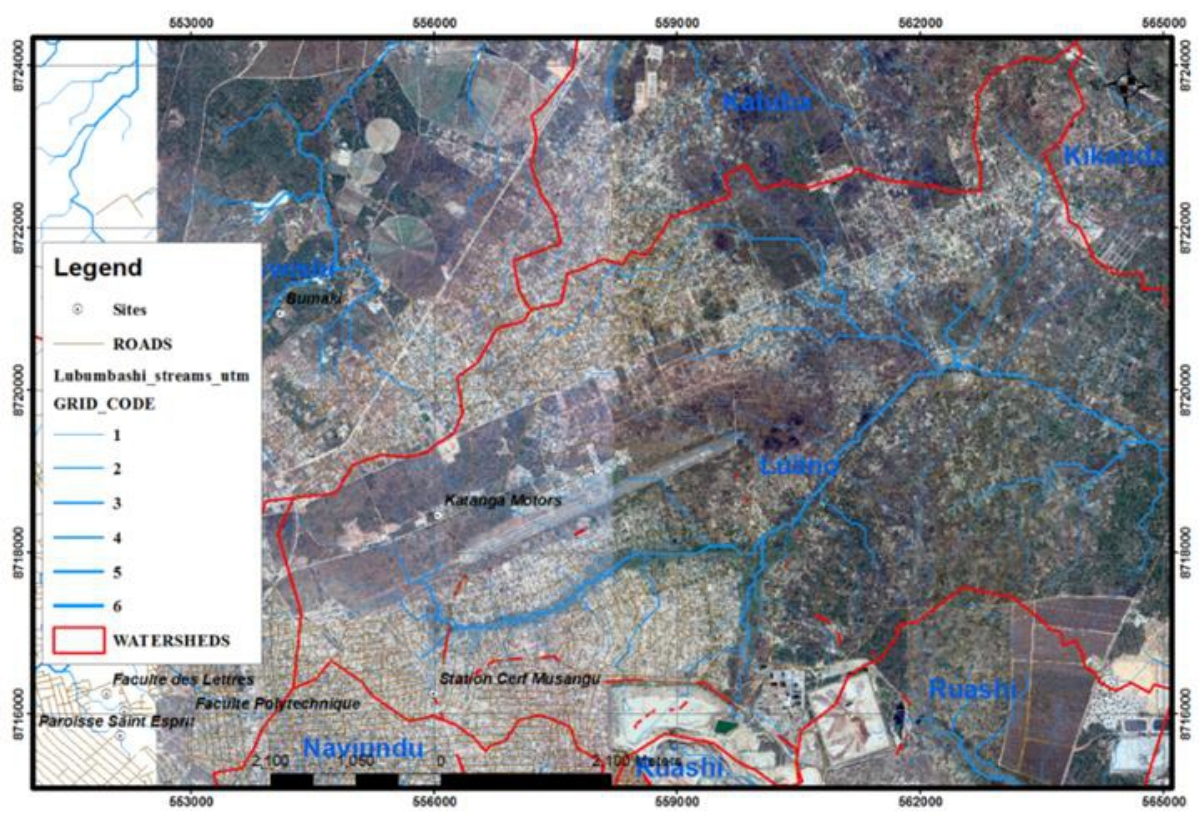

Figure 1 : Localisation de l'aéroport de la Luano par rapport au sous bassin versant de la Luano

\section{Matériels et Méthodes}

La méthode utilisée dans cet article est celle de l'intelligence artificielle ; approche par deep learning basée sur le fondement biologique et mathématique.

\section{Fondement biologique des réseaux de neurones}

Les neurones biologiques possèdent trois principales composantes :

- Les dendrites ;

- Le corps cellulaire ;

- L'axone.

Les dendrites forment un maillage de récepteurs nerveux qui permettent d'acheminer vers le corps du neurone des signaux électriques en provenance d'autres neurones. Celui-ci agit comme une espèce d'intégrateur en accumulant des charges électriques. Lorsque le neurone devient suffisamment excité (lorsque la charge accumulée dépasse un certain seuil) 
par un processus électrochimique, il engendre un potentiel électrique qui se propage à travers son axone pour éventuellement venir exciter d'autres neurones. Le point de contact entre l'axone d'un neurone et le dendrite d'un autre neurone est appelé la synapse. C'est l'arrangement spatial de neurones et de leur axone, ainsi que la qualité des connexions synaptiques individuelles qui détermine la fonction précise d'un réseau de neurone biologique.

\section{Fondement mathématique des réseaux de neurones}

Le modèle mathématique d'un neurone artificiel illustré à la figure 2, est essentiellement constitué d'un intégrateur qui effectue la somme pondérée de ses entrées. Le résultat $n$ de cette somme est ensuite transformé par une fonction de transfert $f$ qui produit la sortie $a$ du neurone. Les $R$ entrées du neurone correspondent au vecteur $\overrightarrow{\boldsymbol{p}}=\left[\boldsymbol{p}_{1} \boldsymbol{p}_{2} \ldots \boldsymbol{p}_{R}\right]^{T}$, alors que $\overrightarrow{\boldsymbol{w}}=$ $\left[\boldsymbol{w}_{1,1} \boldsymbol{w}_{1,2} \ldots \boldsymbol{w}_{1, R}\right]^{T}$ représente le vecteur de poids du neurone.

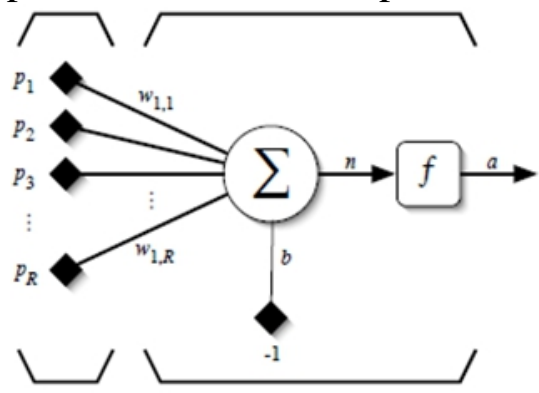

Figure 2 : Schématisation mathématique du modèle neuronal

La sortie $n$ de l'intégrateur est donnée par les équations ci-dessous :

$$
\vec{n}=\sum_{j=1}^{R} w_{1, j} p_{j}-b
$$

$$
\vec{n}=w_{1,1} p_{1}+w_{1,2} p_{2}+\ldots+w_{1, R} p_{R}
$$

$$
\overrightarrow{\boldsymbol{n}}=\overrightarrow{\boldsymbol{w}}^{\boldsymbol{T}} \boldsymbol{p}-\boldsymbol{b}: \text { notation matricielle }
$$

$$
\overrightarrow{\boldsymbol{a}}=\boldsymbol{f}(\boldsymbol{n})=\boldsymbol{f}(\boldsymbol{W} \overrightarrow{\boldsymbol{p}}-\boldsymbol{b}) \text {, avec } \boldsymbol{W} \text { une matrice }
$$


Avec $\boldsymbol{b}$ le biais ou seuil d'activation du neurone.

Cette sortie correspond à une somme pondérée des poids et des entrées moins le biais ou seuil d'activation $\vec{b}$ du neurone. Le résultat $\vec{n}$ de la somme pondérée s'appelle le niveau d'activation du neurone. Lorsque le niveau d'activation $\vec{n}$ atteint ou dépasse le seuil $\vec{b}$, l'argument de $f$ dévient positif ou nul; sinon il est négatif.

Différentes fonctions de transfert peuvent être utilisées comme fonction d'activation du neurone. Les trois fonctions plus utilisées sont : la fonction seuil, la fonction linéaire et la fonction sigmoïde.

La fonction seuil applique un seuil sur son entrée. Lorsqu'une entrée négative ne passe pas le seuil, la fonction retourne alors la valeur de 0 (faux), alors qu'une entrée positive ou nulle dépasse le seuil et la fonction retourne 1 (vrai). Ceci permet de prendre des décisions binaires.

La fonction linéaire affecte directement son entrée à sa sortie. La sortie du neurone correspond à son niveau d'activation.

La fonction sigmoïde ressemble soit à la fonction seuil, soit à la fonction linéaire, selon que l'on est loin ou près de $\vec{b}$ respectivement.

La fonction tangente hyperbolique est une version symétrique de la sigmoïde.

L'apprentissage par réseau de neurones artificiels utilisé dans cet article est supervisé. Celle-ci nécessite l'obtention d'une série des données d'entrée sur lesquelles on connait les sorties correspondantes. Ceci a nécessité la mise en place d'un programme informatique pour réaliser la classification AASHTO des données d'entrainement. La figure 3 présente son interface graphique. Ce programme a été monté grâce à différentes fonctions et classes dans le langage python. Le tableau 1 donne la structuration des données d'entrainement et de test du modèle neuronal.

Tableau 1 : Structuration des données d'entrainement et de test du modèle neuronal

\begin{tabular}{|c|c|c|c|c|c|}
\hline Ip & $\boldsymbol{\omega}_{\boldsymbol{l}}$ & $\boldsymbol{X}_{\mathbf{7 5} \boldsymbol{\mu}}$ & $\boldsymbol{X}_{\mathbf{2 m m}}$ & $\boldsymbol{X}_{\mathbf{4 . 7 5 m m}}$ & AASHTO \\
\hline 15 & 20.53 & 13.60 & 20.60 & 46.80 & A-1-a \\
\hline
\end{tabular}

Ip : indice de plasticité ;

$\boldsymbol{\omega}_{l}:$ limite de liquidité ;

$\boldsymbol{X}_{\mathbf{7 5 \mu} \boldsymbol{\mu}}$ : passants au tamis de 75 micron ou 80 micron suivant la norme utilisée ;

$\boldsymbol{X}_{\mathbf{2 m m}}$ : passants au tamis de $2 \mathrm{~mm}$;

$\boldsymbol{X}_{\mathbf{4 . 7 5 m m}}$ : passants au tamis de $4.75 \mathrm{~mm}$; AASHTO : classe des sols suivant la classification AASHTO. 


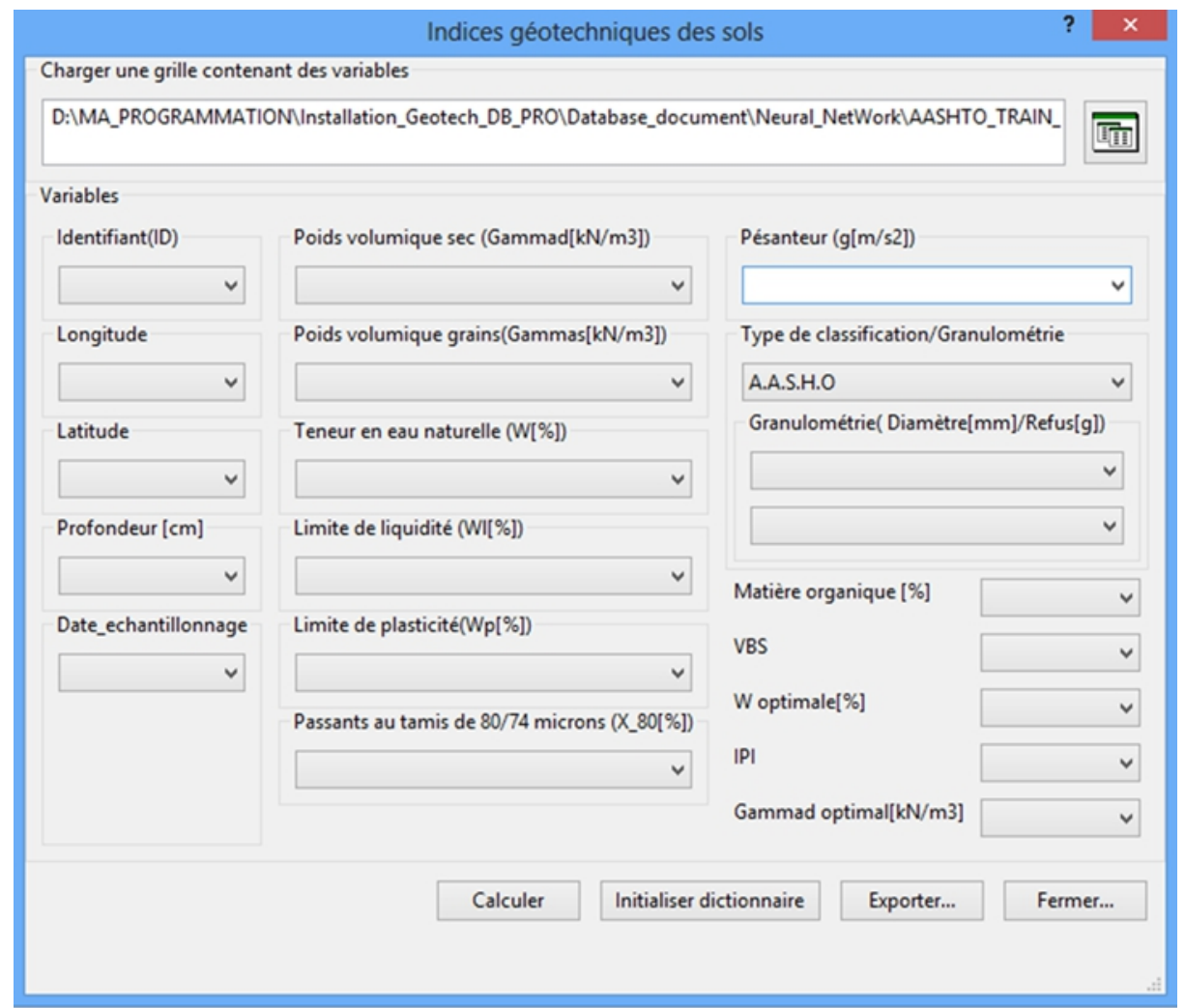

Figure 3 : Interface graphique du programme utilisant l'algorithme AASHTO

Les variables d'entrée du réseau de neurones sont (5 entrées) :

- $\boldsymbol{\omega}_{\boldsymbol{l}}$ : la limite de liquidité;

- $\boldsymbol{I}_{\boldsymbol{p}}$ : l'indice de plasticité;

- $\boldsymbol{X}_{\mathbf{7 5 m i c r o n}}$ : le passant au tamis de $75 \mu \mathrm{m}$ dans la norme ASTM ou $80 \mu m$ dans la norme AFNOR;

- $\boldsymbol{X}_{\mathbf{2 m m}}$ : le passant au tamis de $2 \mathrm{~mm}$;

- $\boldsymbol{X}_{\mathbf{4 . 7 5 m m}}$ : le passant au tamis de $4.75 \mathrm{~mm}$.

Les sorties du réseau de neurones correspondent aux noms des classes géotechniques (12 sorties) :

- A-1-a : les graviers ;

- A-1-b : les graviers ;

- A-3 : les sables fins ;

- A-2-4 : le mélange sables graviers, limons ;

- A-2-5 : le mélange sables graviers, limons ;

- A-2-6 : le mélange sables graviers, argiles ;

- A-2-7 : le mélange sables graviers, argiles ;

- A-4 : les limons ; 
- A-5 : les limons ;

- A-6 : les argiles ;

- A-7-5 : les argiles ;

- A-7-6 : les argiles.

La classe A-8 n'a pas été considérée car elle est appréciée directement sur le terrain en fonction de la quantité en matières organiques. Cette classe des sols n'est pas intéressante pour la mise en place d'ouvrages de génie civil.

Parmi toutes les architectures utilisées en deep learning, le "Multi Layer Perceptron (MLP)" a été considéré comme il s'agit d'un problème de classification (Gulli et al, 2017 ; Chollet, 2018).

Dans la plupart d'architectures, l'apprentissage se traduit par une modification de l'efficacité synaptique, c'est-à-dire par un changement dans la valeur des poids qui relient les neurones d'une couche à l'autre.

Plusieurs algorithmes d'apprentissage sont déjà disponibles dans le framework Keras : SGD (Stochastic Gradient Descent), Adadelta (Adaptative learning rate method), Adagrad (Adaptative gradient), Adam (méthode d'optimisation stochastique), Adamax (Adaptative max), Nadam (Nesterov Adam), RMSprop (Root Mean Square). C'est l'algorithme d'optimisation RMSprop qui a été utilisé.

L'évaluation de la sortie du réseau de neurones entraine la fonction de perte qui mesure le désacord entre la sortie désirée et celle donnée par le réseau. Keras utilise différentes fonctions d'erreur :

- binary cross entropy: C'est la fonction de perte récommandée en classification binaire. La couche de sortie a une simple unité avec la fonction sigmoïde comme fonction d'activation;

- cross entropy: Cette fonction d'erreur est recommandée dans la multiclassification. Dans ce cas la couche de sortie a plusieurs unités avec la fonction d'activation "softmax" (une probabilité est affectée à chacune des classes). C'est cette fonction de perte qui a été utilisée ici;

- squared error: Celle-ci est utilisée dans les problèmes de regression. La couche de sortie a une simple unité.

Plusieurs frameworks sont utilisés pour implémenter les réseaux de neurones artificiels, entre autre: Matlab, Statistica, Caffe, Deeplearning4j, Theano, Tensorflow, Keras, Pybrain. C'est Keras+Theano qui a été retenu pour cet article. Ce framework et le langage de python présentent une documentation très fournie (Swinnen, 2005 ; Rappin et al., 2006 ; Copeland, 2008 ; SciPy-community, 2008; Dupre, 2010 ; Gillies, 2013 ; PostgreSQLGlobal-Development-Group, 2014; PostGIS-Development-Group, 2016 ; Mckinney et al., 2014 ; PySAL-Developers, 2015 ; Lemoine, 2015 ; Hunter et al., 2016 ; Ketkar, 2017 ; Gulli et al., 2017). 


\section{Résultats et discussions}

\section{Modèle de neurones implémenté}

Les modèles suivants ont été testés:

1) Modèle I : nombre d'entrées $5\left(I_{p}, \omega_{l}, X_{75 \mu}, X_{2 m m}, X_{475 m m}\right)$, nombre de sorties 12 (A-1-a, A-1-b, A-2-4, A-2-5, A-2-6, A-2-7, A-4, A-5, A-6, A7-5 et A-7-6), nombre de couches cachées 0 (figure 3);

2) Modèle II et III : nombre d'entrées $5\left(I_{p}, \omega_{l}, X_{75 \mu}, X_{2 m m}, X_{475 m}\right)$, nombre de sorties 12 (A-1-a, A-1-b, A-2-4, A-2-5, A-2-6, A-2-7, A-4, A5, A-6, A-7-5 et A-7-6), nombre de couches cachées 1 (figure 4 et 5);

3) Modèle IV : nombre d'entrées $5\left(I_{p}, \omega_{l}, X_{75 \mu}, X_{2 m m}, X_{475 m m}\right)$, nombre de sorties 12 avec leurs identifiants respectifs (A-1-a, A-1-b, A-3, A-2-4, A-2-5, A-2-6, A-2-7, A-4, A-5, A-6, A-7-5 et A-7-6), nombre de couches cachées 2 (figure 6);

4) Modèle $\mathbf{V}$ : nombre d'entrées $5\left(I_{p}, \omega_{l}, X_{75 \mu}, X_{2 m m}, X_{475 m m}\right)$, nombre de sorties 12 avec leurs identifiants respectifs (A-1-a, A-1-b, A-3, A-2-4, A-2-5, A-2-6, A-2-7, A-4, A-5, A-6, A-7-5 et A-7-6), nombre de couches cachées 7 ( 2 couches de 4 neurones, 2 couches de 7 neurones et 3 couches de 12 neurones).

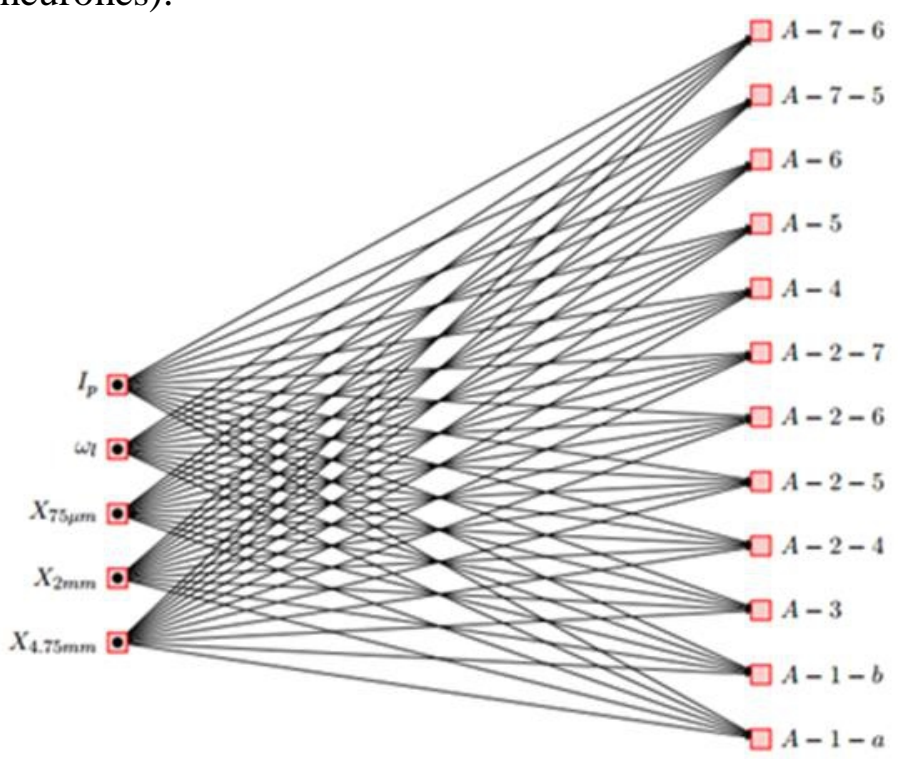

Figure 4 : Modèle du réseau de neurones pour la classification AASHTO sans couche cachée (ce modèle a donné une performance médiocre : 15\%) 


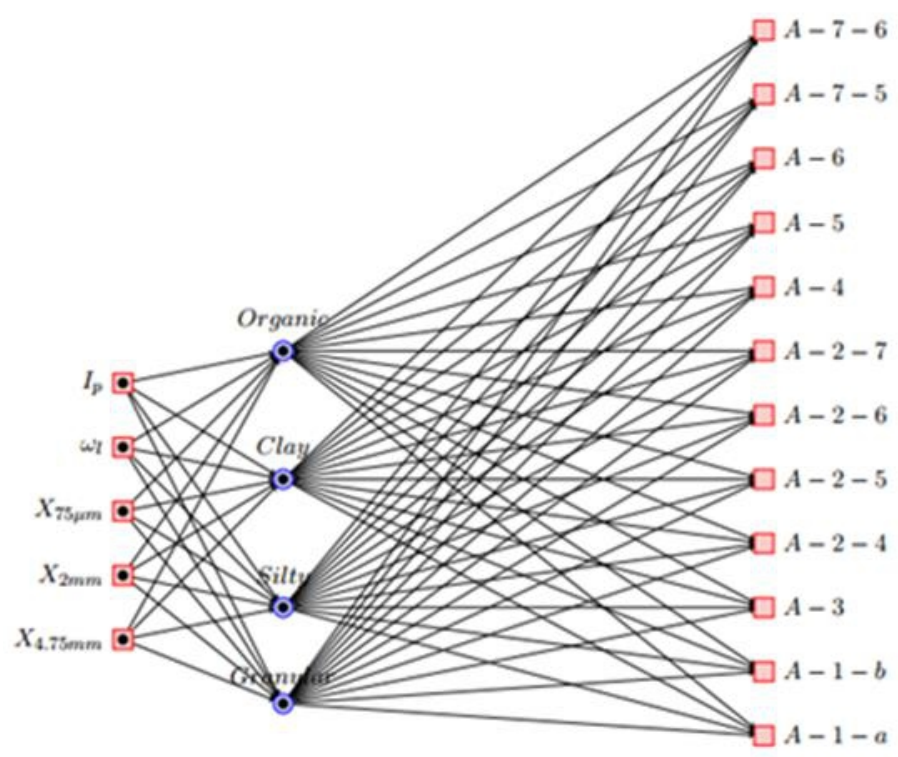

Figure 5 : Modèle du réseau de neurones pour la classification AASHTO à une couche cachée de 4 neurones (comme le modèle précédent, celui-ci a conduit à une performance de $37 \%)$

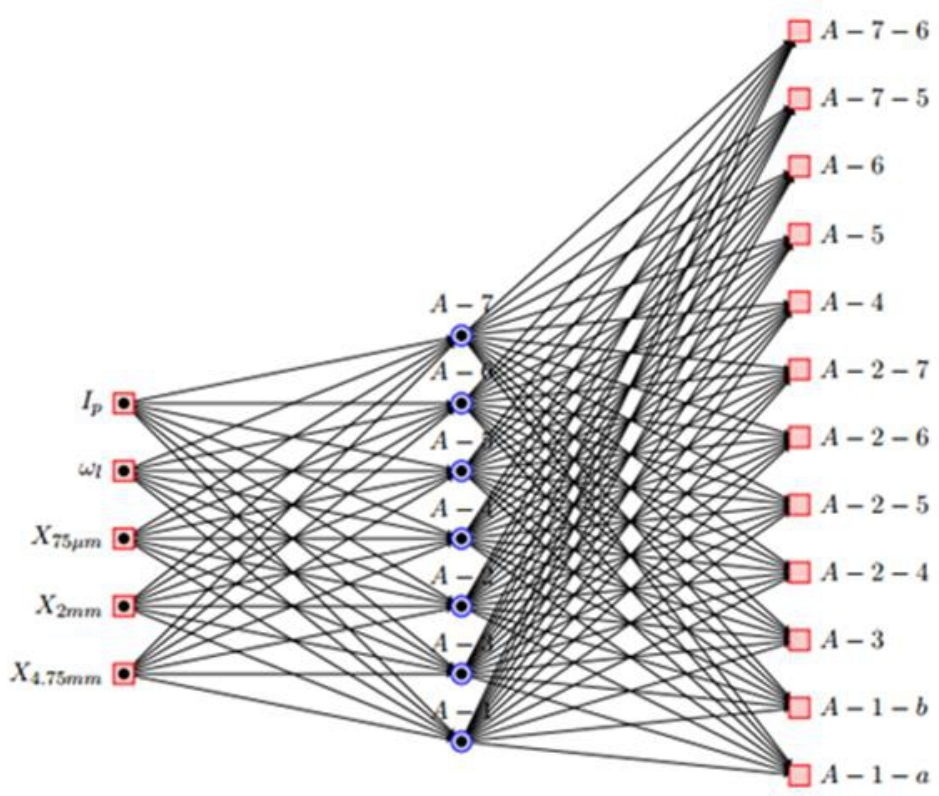

Figure 6 : Modèle du réseau de neurones pour la classification AASHTO à une couche cachée de 7 neurones (ce modèle a donné une performance de 63\%) 


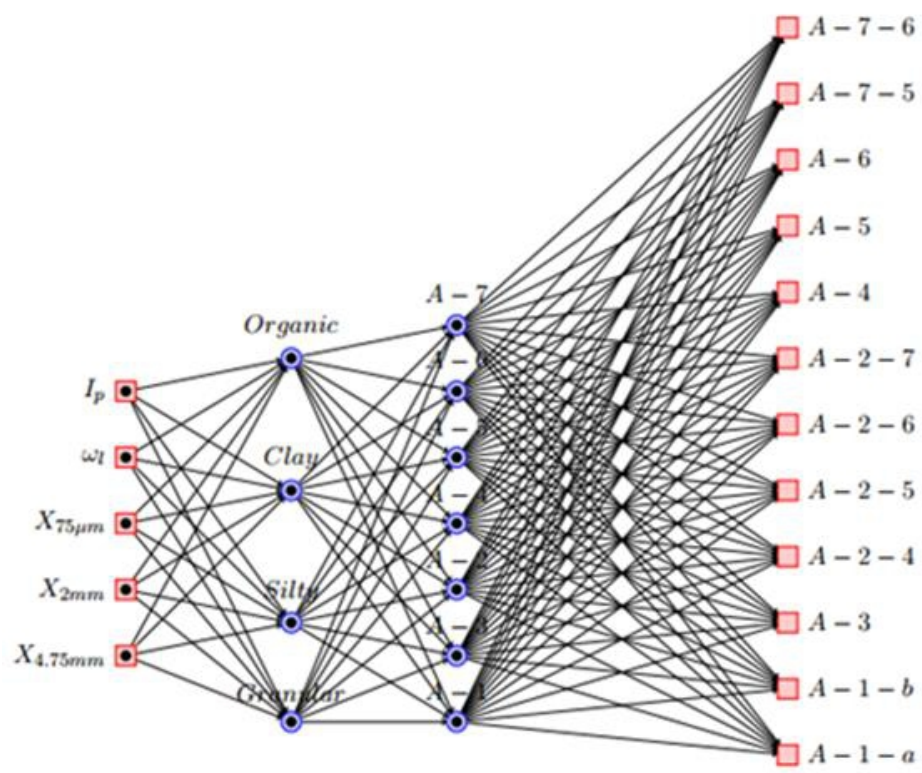

Figure 7 : Modèle du réseau de neurones pour la classification AASHTO à deux couches cachées (ce modèle est le plus optimisé par augmentation des couches cachées et a donné une performance de $86 \%: 86 \%$ de bonnes classifications et $14 \%$ seulement des mauvaises)

Seul le modèle $\mathrm{V}$ a été retenu, car il présente une performance raisonable (86\%) en utilisant les éléments du réseau neuronal suivants :

- Couches cachées avec la fonction d'activation "relu" : 7 ;

- Paramètres dans la couche en entrée : 5 ;

- Classes en sortie avec une fonction d'activation "softmax" : 12 ;

- Algorithme d'optimisation : "rmsprop" ;

- Fonction de perte : "categorical-crossentropy" ;

- Métrique : "categorical-accuracy" ;

- Groupage : 64 ;

- Nombre d'itération : 100.

\section{Apprentissage et validation}

Parmi les propriétés désirables d'un réseau de neurones, la plus fondamentale est la capacité d'apprendre de son environnement, d'améliorer sa performance à travers un processus d'apprentissage. Parizeau (2004) de son côté définit l'apprentissage comme un processus dynamique et itératif permettant de modifier les paramètres d'un réseau en réaction avec les stimuli qu'il reçoit de son environnement. Le type d'apprentissage dépend de la manière dont les changements de paramètres surviennent.

Certaines données ont été utilisées dans la phase de validation pour généraliser le réseau neuronal. Une performance de $86 \%$ a été atteinte. 
Tests et application à la cartographie géotechnique de proximité de l'aéroport international de Luano - Lubumbashi

Pour évaluer le réseau de neurone avec des poids et biais trouvés dans la phase d'apprentissage, nous récourons à la phase de test. Une performance de $86 \%$ a été atteinte, soit $86 \%$ des bonnes classifications et $14 \%$ des divergences (tableau 5 et 6 ).

Tableau 5 : Test de classification algorithmique et neuronale des sols de l'activité 8 dans USDA (1987)

\begin{tabular}{|l|l|r|r|r|r|r|r|r|r|r|r|r|r|r|}
\hline Thématique & A A SHTO & A-1-a & A-1-b & A-2-4 & A-2-5 & A-2-6 & A-2-7 & A-3 & A-4 & A-5 & A-6 & A-7-5 & A-7-6 & P rob \\
\hline Classification dans USDA & A-1-a(2) & 1 & 0 & 0 & 0 & 0 & 0 & 0 & 0 & 0 & 0 & 0 & 0 & 1 \\
\hline Classification dans USDA & A-7-6(27) & 0 & 0 & 0 & 0 & 0 & 0 & 0 & 0 & 0 & 0 & 0 & 1 \\
\hline Classification dans USDA & A-1-a(0) & 1 & 0 & 0 & 0 & 0 & 0 & 0 & 0 & 0 & 0 & 0 & 0 & 1 \\
\hline Classification dans USDA & A-7-5(9) & 0 & 0 & 0 & 0 & 0 & 0 & 0 & 0 & 0 & 0.11 & 0.45 & 0.44 & 1 \\
\hline Classification dans USDA & A-1-b(1) & 0 & 0 & 0 & 0 & 0 & 0 & 1 & 0 & 0 & 0 & 0 & 0 & 1 \\
\hline Classification dans USDA & A-7-6(19) & 0 & 0 & 0 & 0 & 0 & 0 & 0 & 0 & 0 & 0.05 & 0.14 & 0.81 \\
\hline Classification dans USDA & A-5(10) & 0 & 0 & 0 & 0 & 0 & 0 & 0 & 0.01 & 0.97 & 0 & 0.02 & 0 \\
\hline Classification dans USDA & A-1-b(0) & 0 & 0.04 & 0.05 & 0 & 0 & 0 & 0 & 0.9 & 0 & 0 & 0 & 0 \\
\hline Classification dans USDA & A-4(0) & 0 & 0.03 & 0.01 & 0 & 0 & 0 & 0 & 0.96 & 0 & 0 & 0 & 0 \\
\hline Classification dans USDA & A-2-5(0) & 0 & 0.9 & 0 & 0 & 0 & 0 & 0 & 0.09 & 0 & 0 & 0 & 0 \\
\hline Classification dans USDA & A-2-7(0) & 0 & 0 & 0 & 0.01 & 0.01 & 0.71 & 0 & 0 & 0.26 & 0 & 0 & 0 \\
\hline Classification dans USDA & A-2-6(0) & 0 & 0 & 0 & 0.16 & 0.49 & 0.34 & 0 & 0 & 0.01 & 0 & 0 & 0 \\
\hline
\end{tabular}


Tableau 6 : Test de classification algorithmique et neuronale des sols de proximité de l'aéroport international de Luano

\begin{tabular}{|c|c|c|c|c|c|c|c|c|c|c|c|c|c|c|}
\hline Thématique & AASHTO & A-1-a & $A-1-b$ & A-2-4 & A-2-5 & A-2-6 & A-2-7 & $A-3$ & A-4 & A-5 & A-6 & A-7-5 & A-7-6 & Prob \\
\hline Latérite & $A-1-b(0)$ & 0.00 & 0.99 & 0.00 & 0.00 & 0.00 & 0.00 & 0.00 & 0.01 & 0.00 & 0.00 & 0.00 & 0.00 & 1.00 \\
\hline Latérite & $-2-4(0)$ & 0.00 & 0.00 & 0.90 & 0.00 & 0.10 & 0.00 & 0.00 & 0.00 & 0.00 & 0.00 & 0.00 & 0.00 & 1.00 \\
\hline Latérite & A-2-4(0) & 0.00 & 0.00 & 0.94 & 0.00 & 0.04 & 0.00 & 0.00 & 0.00 & 0.02 & 0.00 & 0.00 & 0.00 & 1.00 \\
\hline Latérite & A-2-6(1) & 0.00 & 0.00 & 0.04 & 0.00 & 0.95 & 0.00 & 0.00 & 0.00 & 0.00 & 0.00 & 0.00 & 0.00 & 1.00 \\
\hline Latérite & A-2-4(0) & 0.00 & 0.00 & 0.97 & 0.00 & 0.03 & 0.00 & 0.00 & 0.00 & 0.00 & 0.00 & 0.00 & 0.00 & 1.00 \\
\hline Latérite & A-2-4(0) & 0.00 & 0.00 & 0.97 & 0.00 & 0.03 & 0.00 & 0.00 & 0.00 & 0.00 & 0.00 & 0.00 & 0.00 & 1.00 \\
\hline Latérite & A-2-4(0) & 0.00 & 0.00 & 0.90 & 0.00 & 0.10 & 0.00 & 0.00 & 0.00 & 0.00 & 0.00 & 0.00 & 0.00 & 1.00 \\
\hline Latérite & $A-1-b(0)$ & 0.00 & 0.95 & 0.04 & 0.00 & 0.00 & 0.00 & 0.00 & 0.00 & 0.00 & 0.00 & 0.00 & 0.00 & 1.00 \\
\hline Latérite & A-2-4(0) & 0.00 & 0.80 & 0.18 & 0.00 & 0.00 & 0.00 & 0.00 & 0.02 & 0.00 & 0.00 & 0.00 & 0.00 & 1.00 \\
\hline Latérite & A-2-4(0) & 0.00 & 0.51 & 0.02 & 0.00 & 0.00 & 0.00 & 0.00 & 0.47 & 0.00 & 0.00 & 0.00 & 0.00 & 1.00 \\
\hline Nid de poule & $A-6(6)$ & 0.00 & 0.00 & 0.00 & 0.00 & 0.00 & 0.00 & 0.00 & 0.00 & 0.00 & 0.98 & 0.00 & 0.01 & 1.00 \\
\hline Nid de poule & A-6(9) & 0.00 & 0.00 & 0.00 & 0.00 & 0.00 & 0.03 & 0.00 & 0.00 & 0.00 & 0.01 & 0.00 & 0.95 & 1.00 \\
\hline Nid de poule & A-7-6(33) & 0.00 & 0.00 & 0.00 & 0.00 & 0.00 & 0.00 & 0.00 & 0.00 & 0.00 & 0.00 & 0.00 & 1.00 & 1.00 \\
\hline Nid de poule & $A-4(0)$ & 0.00 & 0.01 & 0.01 & 0.00 & 0.00 & 0.00 & 0.00 & 0.97 & 0.02 & 0.00 & 0.00 & 0.00 & 1.00 \\
\hline Nid de poule & $A-6(6)$ & 0.00 & 0.00 & 0.03 & 0.00 & 0.00 & 0.00 & 0.00 & 0.25 & 0.00 & 0.72 & 0.00 & 0.00 & 1.00 \\
\hline Nid de poule & $A-4(6)$ & 0.00 & 0.00 & 0.04 & 0.00 & 0.00 & 0.00 & 0.00 & 0.85 & 0.01 & 0.09 & 0.00 & 0.00 & 1.00 \\
\hline Nid de poule & A-6(10) & 0.00 & 0.00 & 0.00 & 0.00 & 0.00 & 0.00 & 0.00 & 0.00 & 0.00 & 0.68 & 0.00 & 0.31 & 1.00 \\
\hline Nid de poule & $A-6(7)$ & 0.00 & 0.00 & 0.02 & 0.00 & 0.00 & 0.00 & 0.00 & 0.27 & 0.03 & 0.61 & 0.01 & 0.06 & 1.00 \\
\hline Nid de poule & $A-4(4)$ & 0.00 & 0.00 & 0.01 & 0.00 & 0.00 & 0.00 & 0.00 & 0.93 & 0.06 & 0.00 & 0.00 & 0.00 & 1.00 \\
\hline Nid de poule & A-6(18) & 0.00 & 0.00 & 0.00 & 0.00 & 0.00 & 0.00 & 0.00 & 0.00 & 0.00 & 0.32 & 0.00 & 0.68 & 1.00 \\
\hline Nid de poule & $A-4(0)$ & 0.00 & 0.18 & 0.35 & 0.00 & 0.00 & 0.00 & 0.00 & 0.47 & 0.00 & 0.00 & 0.00 & 0.00 & 1.00 \\
\hline Nid de poule & $A-6(3)$ & 0.00 & 0.00 & 0.08 & 0.00 & 0.09 & 0.01 & 0.00 & 0.66 & 0.00 & 0.16 & 0.00 & 0.00 & 1.00 \\
\hline Nid de poule & $A-4(4)$ & 0.00 & 0.00 & 0.05 & 0.00 & 0.00 & 0.00 & 0.00 & 0.86 & 0.00 & 0.08 & 0.00 & 0.00 & 1.00 \\
\hline Nid de poule & A-6(9) & 0.00 & 0.00 & 0.00 & 0.00 & 0.00 & 0.00 & 0.00 & 0.00 & 0.00 & 0.48 & 0.00 & 0.51 & 1.00 \\
\hline Nid de poule & $A-6(9)$ & 0.00 & 0.00 & 0.00 & 0.00 & 0.00 & 0.00 & 0.00 & 0.01 & 0.00 & 0.97 & 0.00 & 0.02 & 1.00 \\
\hline Nid de poule & $A-6(21)$ & 0.00 & 0.00 & 0.00 & 0.00 & 0.00 & 0.00 & 0.00 & 0.00 & 0.00 & 0.01 & 0.00 & 0.99 & 1.00 \\
\hline Nid de poule & $A-4(0)$ & 0.00 & 0.08 & 0.21 & 0.00 & 0.00 & 0.00 & 0.00 & 0.70 & 0.00 & 0.00 & 0.00 & 0.00 & 1.00 \\
\hline Nid de poule & $A-4(4)$ & 0.00 & 0.00 & 0.06 & 0.00 & 0.00 & 0.00 & 0.00 & 0.89 & 0.00 & 0.05 & 0.00 & 0.00 & 1.00 \\
\hline Nid de poule & A-6(11) & 0.00 & 0.00 & 0.00 & 0.00 & 0.00 & 0.03 & 0.00 & 0.00 & 0.00 & 0.00 & 0.00 & 0.97 & 1.00 \\
\hline Nid de poule & A-6(13) & 0.00 & 0.00 & 0.00 & 0.00 & 0.00 & 0.00 & 0.00 & 0.00 & 0.00 & 0.02 & 0.00 & 0.98 & 1.00 \\
\hline Nid de poule & A-6(14) & 0.00 & 0.00 & 0.00 & 0.00 & 0.00 & 0.00 & 0.00 & 0.00 & 0.00 & 0.62 & 0.00 & 0.37 & 1.00 \\
\hline Nid de poule & A-6(13) & 0.00 & 0.00 & 0.00 & 0.00 & 0.00 & 0.00 & 0.00 & 0.00 & 0.00 & 0.87 & 0.00 & 0.13 & 1.00 \\
\hline Nid de poule & A-7-6(14) & 0.00 & 0.00 & 0.00 & 0.00 & 0.00 & 0.00 & 0.00 & 0.00 & 0.00 & 0.05 & 0.35 & 0.60 & 1.00 \\
\hline Nid de poule & $A-4(1)$ & 0.00 & 0.00 & 0.01 & 0.00 & 0.00 & 0.00 & 0.00 & 0.69 & 0.30 & 0.00 & 0.00 & 0.00 & 1.00 \\
\hline Nid de poule & A-6(9) & 0.00 & 0.00 & 0.01 & 0.00 & 0.00 & 0.00 & 0.00 & 0.19 & 0.29 & 0.25 & 0.12 & 0.14 & 1.00 \\
\hline Nid de poule & A-7-6(14) & 0.00 & 0.00 & 0.00 & 0.00 & 0.00 & 0.00 & 0.00 & 0.00 & 0.00 & 0.01 & 0.61 & 0.37 & 1.00 \\
\hline Nid de poule & A-6(11) & 0.00 & 0.00 & 0.00 & 0.00 & 0.00 & 0.00 & 0.00 & 0.00 & 0.14 & 0.03 & 0.70 & 0.13 & 1.00 \\
\hline Nid de poule & $A-4(6)$ & 0.00 & 0.00 & 0.01 & 0.00 & 0.00 & 0.00 & 0.00 & 0.51 & 0.48 & 0.00 & 0.00 & 0.00 & 1.00 \\
\hline Nid de poule & $A-4(7)$ & 0.00 & 0.00 & 0.01 & 0.00 & 0.00 & 0.00 & 0.00 & 0.53 & 0.44 & 0.01 & 0.00 & 0.00 & 1.00 \\
\hline Termitiè re & $A-4(4)$ & 0.00 & 0.00 & 0.04 & 0.00 & 0.00 & 0.00 & 0.00 & 0.93 & 0.01 & 0.03 & 0.00 & 0.00 & 1.00 \\
\hline Termitiè re & A-6(11) & 0.00 & 0.00 & 0.00 & 0.00 & 0.00 & 0.00 & 0.00 & 0.03 & 0.03 & 0.51 & 0.12 & 0.31 & 1.00 \\
\hline Termitiè re & A-6(10) & 0.00 & 0.00 & 0.01 & 0.00 & 0.00 & 0.00 & 0.00 & 0.16 & 0.43 & 0.14 & 0.15 & 0.11 & 1.00 \\
\hline Termitiè re & A-6(12) & 0.00 & 0.00 & 0.00 & 0.00 & 0.00 & 0.00 & 0.00 & 0.00 & 0.01 & 0.32 & 0.20 & 0.47 & 1.00 \\
\hline Termitiè re & A-7-6(16) & 0.00 & 0.00 & 0.00 & 0.00 & 0.00 & 0.00 & 0.00 & 0.00 & 0.00 & 0.16 & 0.03 & 0.81 & 1.00 \\
\hline Termitiè re & $A-6(9)$ & 0.00 & 0.00 & 0.01 & 0.00 & 0.00 & 0.00 & 0.00 & 0.26 & 0.56 & 0.07 & 0.06 & 0.04 & 1.00 \\
\hline Termitiè re & $A-6(8)$ & 0.00 & 0.00 & 0.01 & 0.00 & 0.00 & 0.00 & 0.00 & 0.17 & 0.02 & 0.72 & 0.01 & 0.07 & 1.00 \\
\hline Termitiè re & $A-4(8)$ & 0.00 & 0.00 & 0.00 & 0.00 & 0.00 & 0.00 & 0.00 & 0.08 & 0.89 & 0.00 & 0.02 & 0.00 & 1.00 \\
\hline Termitiè re & A-6(13) & 0.00 & 0.00 & 0.00 & 0.00 & 0.00 & 0.00 & 0.00 & 0.00 & 0.13 & 0.00 & 0.81 & 0.05 & 1.00 \\
\hline Termitiè re & A-6(11) & 0.00 & 0.00 & 0.00 & 0.00 & 0.00 & 0.00 & 0.00 & 0.04 & 0.07 & 0.43 & 0.16 & 0.30 & 1.00 \\
\hline
\end{tabular}

Du point de vue géotechnique, deux cartes ont été élaborées :

- La carte de la figure 8 correspondant aux classes AASHTO identifiés à l'aide du logiciel (approche algorithmique) et ;

- Celle de la figure 9 obtenue par la classification neuronale. 


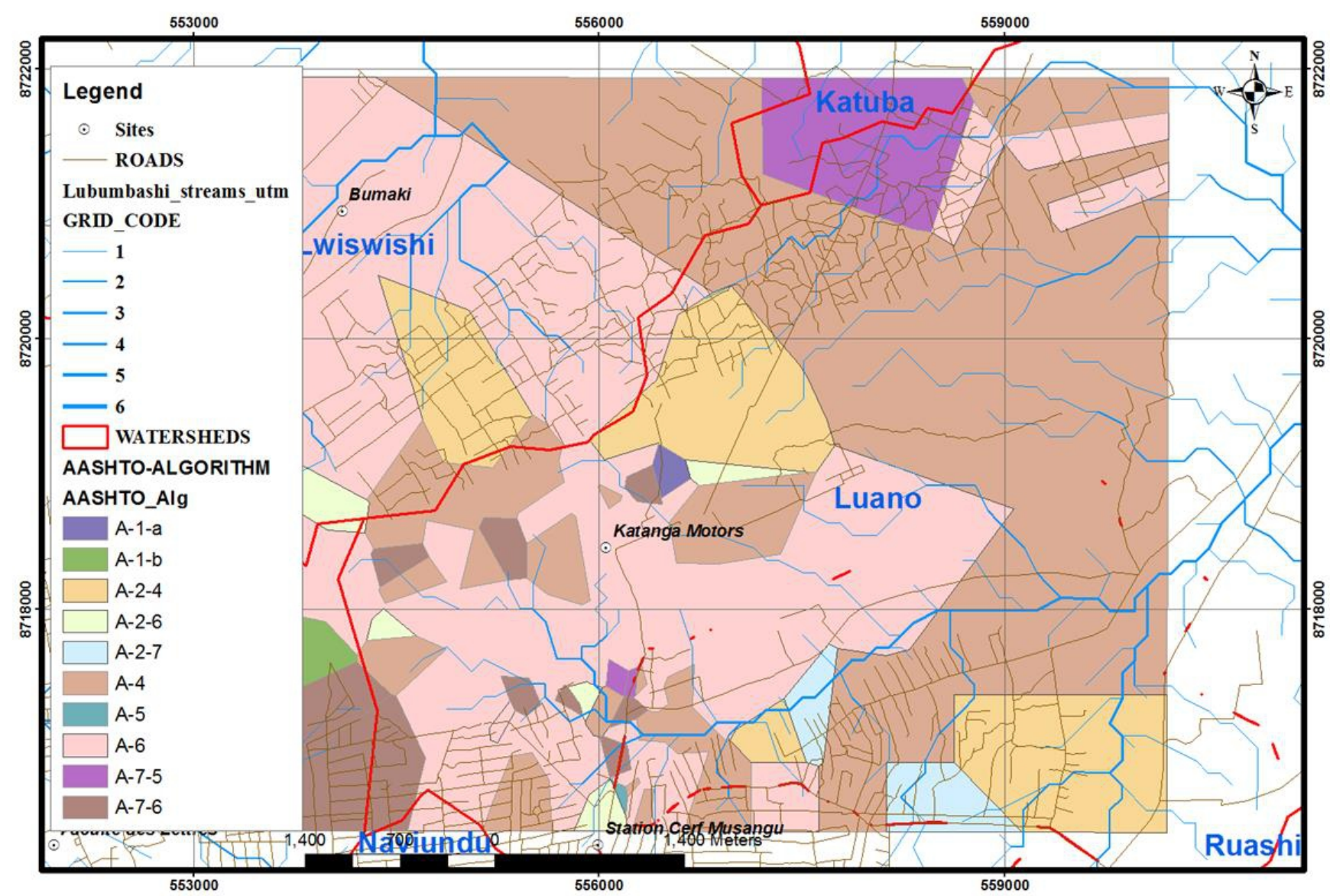

Figure 8 : Carte géotechnique de proximité de l'aéroport international de Luano par algorithme AASHTO 


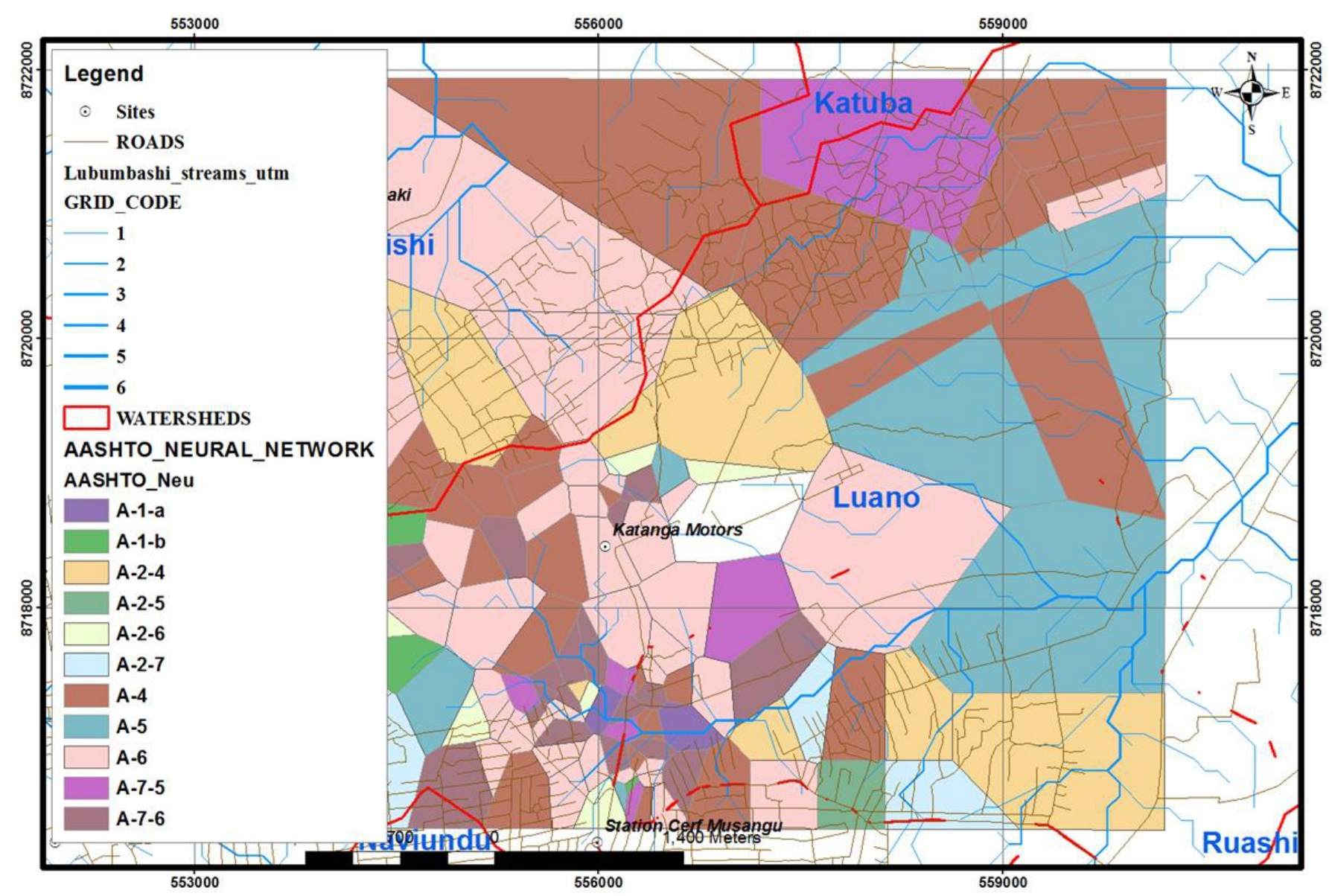

Figure 9 : Carte géotechnique de proximité de l'aéroport international de Luano par Deep Learning 
Ces deux dernières cartes montrent plusieurs points communs (à 86\%). Des divergences sont plus remarquées sur les classes AASHTO ayant l'indice 5 dans le symbole. Ces sols présentent des fortes similitudes avec ceux à indice 4 et 6. Ceci s'explique par le fait qu'ils proviennent souvent de l'altération des sols préexistents. C'est le cas également de certains sols A-7 par rapport aux sols A-6. La méthode par réseau de neurones ressort la classe A-2-5 qui est absente sur la carte obtenue avec l'algorithme AASHTO. Ceci montre la puissance de cette méthode dans la discrimination par similitude des géomatériaux.

\section{Conclusion}

Parmi les nombreux systèmes de classification des géomatériaux, la classification AASHTO a été retenue pour illustrer la méthode : discrimination basée sur l'apprentissage profond (« deep learning»). De toutes les architectures utilisées en pratique, le perceptron multicouche est adapté pour implémenter le modèle neuronal appliqué aux problèmes de classification des géomatériaux.

En comparant les résultats obtenus à l'aide de l'approche algorithmique et ceux de la méthode neuronale, plusieurs points communs sont identifiés (à 86\%). La méthode par réseau de neurones a permis d'identifier certaines classes passées inaperçues avec la classification algorithmique (c'est le cas par exemple de la classe A-2-5). Pour améliorer la performance du réseau afin de discriminer davantage les classes, il fallait jouer sur les éléments suivants:

- la profondeur du réseau: "deep learning";

- le groupage: "batching";

- le nombre d'itérations: "epoch ";

- l'augmentation des données dans la base d'apprentissage;

- l'usage d'un algorithme d'apprentissage approprié au problème à résoudre.

Cette nouvelle approche de classification géotechnique s'avère adaptée aux classifications des géomatériaux utilisées dans les travaux d'ingéniérie civile. Elle donne même des résultats très interessants en se basant seulement sur des photographies numériques prises lors d'une campagne géotechnique. Dans ce dernier cas, les données d'entrée doivent être constituées des pixels.

\section{References:}

1. F. CHOLLET 2018. Deep learning with python. MANNING, 2018. $386 \mathrm{p}$.

2. R. COPELAND 2008. Mapping Python to Database, Essentiel SQLAchemy. O'REILLY, 1er edition, $215 \mathrm{p}$. 
3. M. DAS et K. SOBHAN 2012. Principles of geotechnical engineeting. SI, VIII, edition, 770p.

4. X. DUPRE 2010. Langages Informatiques, Programmation avec le langage Python. Outil commode au service de l'ingénieur. ENSAE, $336 \mathrm{p}$.

5. S. GILLIES 2013. Geoalchemy2 documentation. Release 0.2.6. http://toblerity.org/shapely/manual.html.

6. A. GULLI et S. PAL 2017. Deep learning with keras, implementation neural networks with keras on theano and tensorflow. Packt, 190p.

7. J. HUNTER, D. DALE, E. FIRING, et M. DROETTBOOM 2016. Matplotlib. Release 1.5.1, 2864p.

8. P. KASONGO 2017a. Mise en place d'un système d'informations géotechniques, validation avec des données géotechniques de la ville de Lubumbashi en République Démocratique du Congo. Master's thesis, Université de Lubumbashi, Lubumbashi, 155p.

9. P. KASONGO 2017b. Mise en place d'un système d'informations géotechniques. Editions Universitaires Européennes, Université de Lubumbashi, Lubumbashi, 168p.

10. P. KASONGO, G. MUKOKO, L. KIPATA et J.M. LUNDA 2018. Elaboration de la carte géotechnique de Lubumbashi: guide technique de sélection des sites d'implantation d'ouvrages du génie civil. European Scientific Journal, Vol. 14, N³6.

11. N. KETKAR 2017. Deep learning with python, a hands-on introduction, apress edition, 160p.

12. E. LEMOINE 2015. The shapely user manual, 44p.

13. W. MCKINNEY ET PYDATA DEVELOPMENT TEAM 2014. Pandas - powerful python data analysis toolkit.release 0.15.1. http://github.com/pydata/pandas, $1757 \mathrm{p}$.

14. M. PARIZEAU 2004. Réseaux de neurones, GIF-21140 et GIF-64326. Université Laval, 117p.

15. POSTGIS-DEVELOPMENT-GROUP 2016. PostGIS 2.2.1 Manual. The PostGIS Development Group, 774p.

16. POSTGRESQL-GLOBAL-DEVELOPMENT-GROUP 2014. Documentation PostgreSQL 9.4.0. The PostgreSQL Global Development Group, 2240p.

17. PYSAL-DEVELOPERS 2015. Pysal documentation. Release 1.11.0dev, 503p.

18. N. RAPPIN et R. DUNN 2006. wxPython in action. MANNING, 583p.

19. SCIPY-COMMUNITY 2008. Scipy reference guide. Release 0.7.dev, $435 \mathrm{p}$.

20. L. SOW, F. BERNARD, S. KAMALI-BERNARD et C. M. F. KEBE 2017a. Comportement mécanique de graves à base de MIOM traitées 
au ciment par une modélisation numérique 3D hiérarchique multiéchelle. 35èmes Rencontres de l'AUGC, ECN/UN, 22 au 24 mai 2017, Nantes, France.

21. L. SOW, F. BERNARD, S. KAMALI-BERNARD et C. M. F. KEBE 2017b. Détermination du comportement visco-élastique d'une grave routière à base de MIOM traitée au bitume par modélisation numérique 3D hiérarchique multi-échelles. 35èmes Rencontres de l'AUGC, ECN/UN, 22 au 24 mai 2017, Nantes, France.

22. L. SOW 2018a. Approche couplée expérimentation-modélisation multi-échelle pour la détermination du comportement mécanique des graves routières traitées aux liants. Application à la valorisation des Mâchefers d'Incinération de Déchets Non Dangereux. PhD. Dissertation, INSA Rennes.

23. L. SOW, F. BERNARD, S. KAMALI-BERNARD et C. M. F. KEBE $2018 b$. Experiment-based modelling of the mechanical behaviour of non-hazardous waste incineration bottom ashes treated by hydraulic binder. MATEC Web of Conferences, 149, 01038.

24. L. SOW, F. BERNARD, S. KAMALI-BERNARD et C. M. F. KEBE $2018 \mathrm{c}$. Mesoscale modeling of the temperature-dependent viscoelastic behavior of a Bitumen-Bound Gravels. Coupled Systems Mechanics, 7(5), 509-524.

25. L. SOW, S. KAMALI-BERNARD, O. BARTIER, G. MAUVOISIN, et F. BERNARD 2018d. Experimental Estimation of the Elastic Modulus of Non-Hazardous Waste Incineration Bottom Ash Aggregates by Indentation Tests - Microanalysis of Particles by Scanning Electron Microscopy. Advanced Materials Research, 1145, 80-84.

26. L. SOW, F. BERNARD, S. KAMALI-BERNARD et C. M. F. KEBE $2018 e$. Approche couplée modélisation-expérimentation pour étudier le comportement mécanique de Mâchefers d'Incinération des Déchets Non Dangereux traités avec un liant hydraulique. MATEC Web of Conferences, 149, 01038.

27. L. SOW, S. KAMALI-BERNARD, O. BARTIER, G. MAUVOISIN, et F. BERNARD 2019a. Tests d'indentation instrumentée sur granulats de Mâchefers d'Incinération de Déchets Non Dangereux. Influence de la taille de l'indenteur sur le module élastique. MATEC Web of Conferences, 261, 01002.

28. L. SOW, S. KAMALI-BERNARD, G. MAUVOISIN, O. BARTIER and F. BERNARD 2019b. Original Experimental Campaign of Indentation Instrumented on Aggregates of Non-Hazardous Waste Incineration Bottom Ash to Study the Heterogeneity of their Rigidity. Materials Science Forum (submitted). 
29. L. SOW, F. BERNARD, S. KAMALI-BERNARD and C. M. F. KEBE 2019c. Mechanical behaviour of Cement-Bound Gravels by experiment-based 3D multi-scale modelling: application to Non Hazardous Waste Incineration bottom ashes aggregates for use in road techniques. Submitted to Road Materials and Pavement Design.

30. G. SWINNEN 2005. Apprendre à programmer avec python. Eyrolles, $370 \mathrm{p}$.

31. USDA 1987. Soil mechanics level I, module 2 : AASHTO (American Association of State Highway and Transportation Officials), study guide. Soil Conservative Service, 71p. 